\title{
Correlation between lower limb muscle asymmetry during the sit-to-stand task and spatiotemporal gait asymmetry in subjects with stroke
}

\author{
Sungkwang Ju* \\ Department of Physical Therapy, Kaya University, Gimhae, Korea
}

The purpose of this study was to investigate the relationship between the both lower limb muscle asymmetry during sit-to-stand (STS) and spatiotemporal gait asymmetry in subjects with stroke. Twenty-nine patients were included in this study, lower limb muscle (rectus femoris, tibialis anterior, gastrocnemius) asymmetry during STS, five times sit-tostand (FTSTS) and spatiotemporal (speed, step length, step time, swing time, stance time, single support time) gait asymmetry were measured. According to the results of the measurement, significant positive correlations between gastrocnemius and rectus femoris asymmetry during

\section{INTRODUCTION}

Stroke usually results in hemiparesis or muscle weakness contralateral to the cerebral lesion, increased abnormal muscle tone, and deficit of coordination (Geyh et al., 2004). Muscle impairments on the paretic side are associated with limitations in activities of daily living (ADL), such as walking (Nasciutti-Prudente et al., 2009) and sit-to-stand (STS) (Bohannon, 2007). Asymmetric dynamic posture and movement is the most prevalent locomotor deficit associated with stroke-related hemiparesis (Winstein et al., 1989). Weight-bearing asymmetry has been linked to impaired motor function and the standing balance problems of stroke patients (Pyöriä et al., 2004). Impaired motor function may be a result of muscle weakness or abnormal tone causing difficulty in muscle use and leading to increased postural sway, thus leading to an increased risk of falling (Chitra and Mishra, 2014).

The STS task is considered a necessary condition for upright movement and for performing other important daily activities
STS and step length asymmetry. Furthermore, significant positive correlations between rectus femoris asymmetry during STS and step time asymmetry. In addition, significant negative correlations between the FTSTS and gait speed. This study's results suggested that necessary to apply exercise that the gastrocnemius and rectus femoris symmetry during STS for improving step length and step time symmetry of gait, and necessary to apply exercise that increase STS speed for gait speed.

Keywords: Stroke, Sit-to-stand, Walking, Asymmetry such as locomotion (Bohannon et al., 2008). Thus, STS performance is fundamental for independence (Janssen et al., 2010). The quadriceps considered a primary motor muscle of the STS task because it promotes knee extension and contributes to hip flexion and stability (Khemlani et al., 1999). The tibialis anterior (TA) is important during the initial phases of the STS to stabilize the foot on the ground, the soleus seems to have an important function in postural adjustments at the end of the STS (Lomaglio and Eng, 2005). Electromyography (EMG) activity of the quadriceps, TA, and soleus muscles in the less affected lower limb was higher than that in affected lower limb during STS task in stroke patients (Prudente et al., 2013). Stroke patients exhibit asymmetrical weight bearing during the STS, placing approximately $41.5 \%$ less load on the paretic leg, and this asymmetric pattern affects gait performance (Chou et al., 2003). After stroke, many people have difficulty standing up and walking independently, due to motor impairments such as weakness and poor coordination (de Sousa et al., 2019).

Walking dysfunction is a major problem for many people af-
*Corresponding author: Sungkwang Ju (iD https://orcid.org/0000-0003-3125-812X Department of Physical Therapy, Kaya University, 208 Samgye-ro, Gimhae 50830, Korea

E-mail: jskwang84@hanmail.net

Received: January 17, 2020 / Accepted: February 5, 2020
This is an Open Access article distributed under the terms of the Creative Commons Attribution Non-Commercial License (https://creativecommons.org/licenses/by-nc/4.0/) which permits unrestricted non-commercial use, distribution, and reproduction in any medium, provided the original work is properly cited. 
flicted by stroke (Algurén et al., 2010). Functional gait is an important factor in ADL (Tomoyuki and Shigeru, 2010). The ability to walk is determined by several factors, including balance, motor function, and endurance, and is affecting by impairments in muscle strength, sensory function, and other factors (van de Port et al., 2008). The slow walking speeds commonly observed following stroke are associated with marked temporal and spatial interlimb asymmetries (Patterson et al., 2010). A major characteristic of hemiplegic gait observed in stroke patients is walking asymmetry, which may increase the risk of falls (Yen et al., 2015). Spatiotemporal gait asymmetry was correlated with muscle weakness (Hsu et al., 2003).

Therapists are aware of the importance of STS and walking after stroke, and the relationship between STS ability and walking performance has been investigated in several studies. However, studies on the relationship between STS asymmetry and gait asymmetry are lacking. Therefore, in this study, investigated the relationship between both lower limb muscle asymmetry during STS and spatiotemporal gait asymmetry in subjects who had a stroke.

\section{MATERIALS AND METHODS}

\section{Participants}

This study included 29 patients with stroke. All subjects had been treated to rehabilitation. The inclusion criteria were: (a) diagnosis of cerebral hemorrhage or cerebral infarction, (b) a minimum of 6-month poststroke, (c) able to independently the sit-tostand and the walking, (d) able to understand both verbal and written information during testing procedures. The exclusion criteria included other orthopedic conditions and neurological disorder. Written informed consent was obtained from all participants and all protocols were approved by the Ethics Committee of the Kaya University (Kaya IRB-264).

\section{STS measurement}

STS maneuvers were performed from a chair without an arm rest at $43 \mathrm{~cm}$ in height and $47.5 \mathrm{~cm}$ in depth. STS began when a verbal signal from the tester. EMG activities of the three muscles on the both lower limbs during STS were recorded using surface TeleMyo 2400T (Noraxon Co., Scottsdale, Arizona, AZ, USA) and pairs of silver-silver chloride disposable electrodes with a diameter of $3 \mathrm{~mm}$ (EL 503, Biopac systems Inc., Goleta, CA, USA). The root mean square values of the raw data were calculated for 250 samples. The EMG activity recording period of $5 \mathrm{sec}$. The threes in each lower limb, were positioned over the muscle fibers of the rectus femo- ris $(\mathrm{RF}), \mathrm{TA}$, and the medial gastrocnemius (GCM) of both lower limbs (Hermens et al., 2000). EMG data were normalized to reference voluntary contraction (RVC), the RVC testing was performed in subjects positioned in the quiet standing. The data obtained from each trial were expressed as a percentage of the RVC.

\section{Five times sit-to-stand test}

The five times sit-to-stand (FTSTS) test measured the time taken to complete five repetitions of the STS. The examiner demonstrated the correct technique for performance of the test. Participant was instructed to stand up and sit down as quickly as possible for 5 times, to place participant's hands on lap and do not use them during the procedure. The timing began when the examiner spoke the "go" and stopped once the back touched the backrest. Participants performed three repetitions, a 1-min rest was given between trials to prevent fatigue.

\section{Gait measurement}

Temporal and spatial parameters of gait were collected using the GAITRite (CIR system Inc., Franklin, New Jersey, NJ, USA). GAITRite is an electronic walking mat that has a walking area of $90-\mathrm{cm}$ width and $460-\mathrm{cm}$ length. Data were stored in a personal computer that calculated spatial and temporal parameters using application software. For the walking measurement, an examiner showed an example and let the participants walk. Walking began $2 \mathrm{~m}$ in front of the walking mat upon a verbal signal from the tester. All participants walked 3 times and walking speed, step length, step time, swing time, stance time, and single support time were measured. The average of the three measurements was used for the analysis.

\section{Asymmetry ratio calculation}

This study evaluated the muscle activation (RF, TA, GCM) of both lower limbs during sit-to-stand and the gait parameter (step length, step time, swing time, stance time, and single support time). To compare the asymmetry of the affected and less affected lower limbs, asymmetry ratio values were calculated using the following equation (Hsu et al., 2003):

Asymmetry ratio $=\mid 1$-affected side/less affected side $\mid$.

A larger value of ratio indicates higher asymmetry between the both sides.

\section{Statistical analysis}

Data will analyze using IBM SPSS Statistics ver. 20.0 (IBM Co., Armonk, NY, USA). The general characteristics were analyzed 
descriptive analysis. The correlation between the subjects' the lower limb muscle asymmetry during the STS and spatiotemporal gait asymmetry was analyzed using the Pearson correlation method. The statistical significance was set at $P<0.05$. The data were presented as the mean with standard deviation values.

\section{RESULTS}

\section{General characteristics of the participants}

The research participants included 21 males and eight females whose average age was $52.2 \pm 12.2$ years old, average height was $165.5 \pm 7.0 \mathrm{~cm}$, and average body weight was $65.2 \pm 9.5 \mathrm{~kg}$ (Table 1).

The correlation between lower limb muscle asymmetry during STS and spatiotemporal gait asymmetry

The correlations between lower limb muscle imbalance during the STS and spatiotemporal gait symmetry were analyzed. There were significant positive correlations between GCM asymmetry and step length asymmetry (0.374) and between RF asymmetry and step length asymmetry (0.427). Furthermore, significant positive correlations between RF asymmetry and step time asymmetry (0.781) (Table 2, Fig. 1). In addition, significant negative correlations between the FTSTS and gait speed (-0.409) (Table 2, Fig. 1).

Table 1. General characteristics of the participants $(n=29)$

\begin{tabular}{lc}
\hline Variable & Value \\
\hline Sex, male:female & $21: 8$ \\
Age (yr) & $52.2 \pm 12.2$ \\
Height (cm) & $165.5 \pm 7.0$ \\
Body weight (kg) & $65.2 \pm 9.5$ \\
\hline
\end{tabular}

Values are presented as number or mean \pm standard deviation.

Table 2. The correlation between lower limb muscle asymmetry during STS and spatiotemporal gait asymmetry

\begin{tabular}{lcccccc}
\hline Variable & $\begin{array}{c}\text { Step length } \\
\text { asymmetry }\end{array}$ & $\begin{array}{c}\text { Swing time } \\
\text { asymmetry }\end{array}$ & $\begin{array}{c}\text { Stance time } \\
\text { asymmetry }\end{array}$ & $\begin{array}{c}\text { Step time } \\
\text { asymmetry }\end{array}$ & $\begin{array}{c}\text { Single support time } \\
\text { asymmetry }\end{array}$ & $\begin{array}{c}\text { Speed } \\
\text { GCM asymmetry }\end{array}$ \\
RF asymmetry & $0.374^{*}$ & -0.183 & -0.312 & -0.275 & -0.168 & 0.013 \\
TA asymmetry & $0.427^{*}$ & 0.255 & 0.103 & $0.781^{* *}$ & 0.068 & -0.339 \\
FSTS & 0.182 & 0.215 & 0.223 & 0.042 & 0.156 & -0.279 \\
\hline
\end{tabular}

STS, sit-to-stand; GCM, gastrocnemius; RF, rectus femoris; TA, tibialis anterior; FTSTS, five times sit-to-stand.

${ }^{*} P<0.05$. ${ }^{*} P<0.01$.
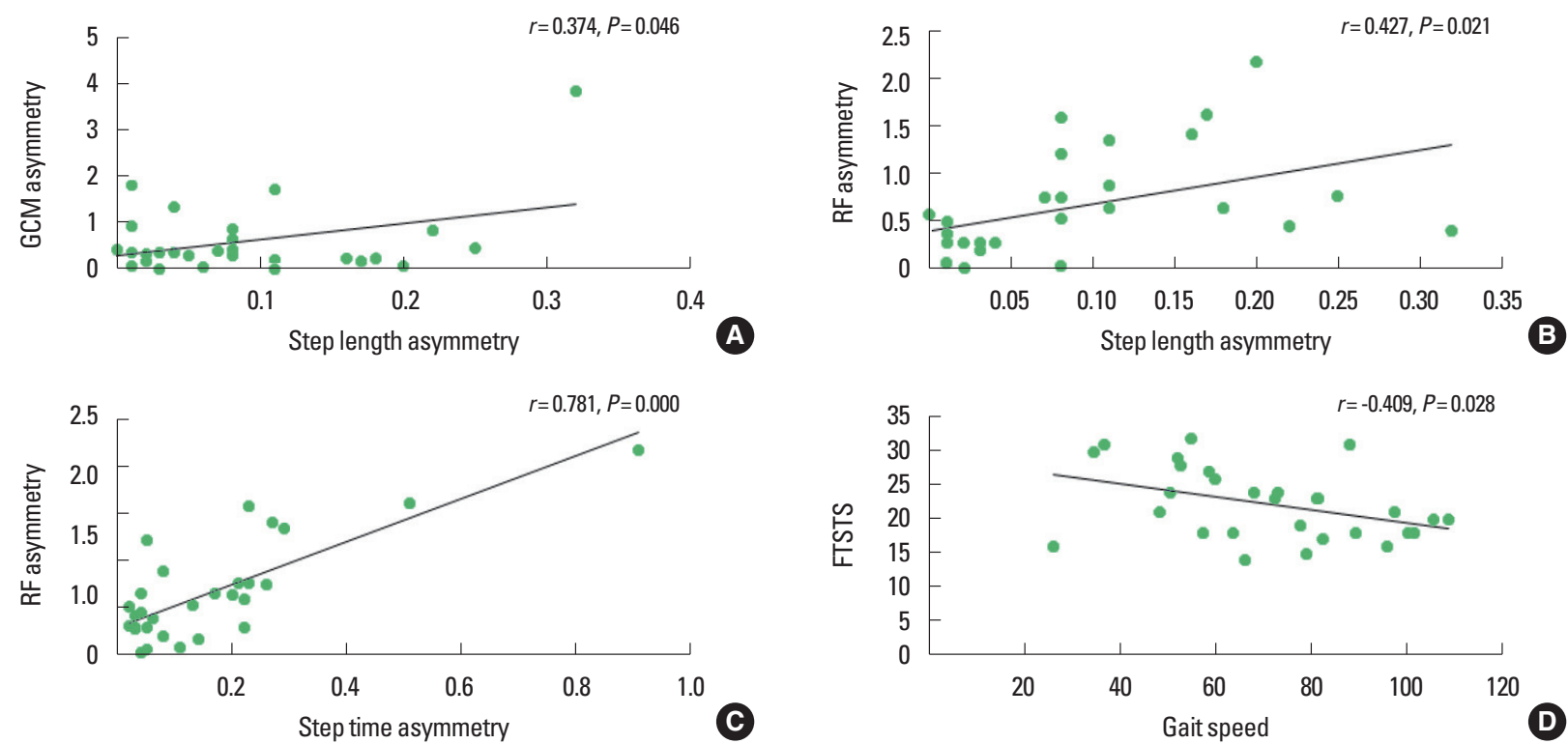

Fig. 1. The correlation between lower limb muscle asymmetry during sit-to-stand and spatiotemporal gait asymmetry: gastrocnemius (GCM) asymmetry and step length asymmetry (A), rectus femoris (RF) asymmetry and step length asymmetry (B), RF asymmetry and step time asymmetry (C), 5 times sit-to-stand (FTSTS) and gait speed (D). 


\section{DISCUSSION}

STS control is closely related to ambulatory ability. After a stroke, the brain's ability to perform the STS becomes asymmetric because of weakness on the affected side and the loss of postural control (Chou et al., 2003). The peak power level required for one to rise from a chair may be related to leg muscle strength and the ability to walk (Cheng et al., 1998). The present study has investigated the relationships between lower limb muscle asymmetry during STS and spatiotemporal gait symmetry in stroke patients. The results of present study found significant correlations between both lower limb muscle asymmetry during STS and spatiotemporal gait asymmetry.

The results of the present study showed positive correlation between GCM and RF asymmetry during STS and step length asymmetry during gait. Quadriceps muscle weakness may be related to loading asymmetry during the STS task (Christiansen et al., 2013), and ankle plantar flexor strength contributes the most to STS performance (McCarthy et al., 2004). Prudente et al. (2013) reported that the significant compensations occur on the nonparetic side instead of the paretic limb during STS task. Lower limb muscle strength and weight-bearing symmetry are related to STS in stroke patients (Lomaglio and Eng, 2005). The results of the present study found that GCM and RF activity are asymmetrical during STS. Although this study measured only part (RF) of the quadriceps and part (GCM) of the plantar flexor, the results are the same as in the previous study. Ankle plantar flexor function is mainly active in the single-leg stance phase during gait (Lamontagne et al., 2002). Allen et al. (2011) reported that patients with poststroke hemiparesis show a common impairment in the ankle plantar flexion moment impulse, but that different compensation mechanisms are utilized by patients according to the pattern of step length asymmetry. In a previous study, patients with a maximal vertical force difference of less than $30 \%$ of the patient's body weight between their legs during STS had better gait performance than other stroke patients (Chou et al., 2003). Considering the present study's results, it seems that the asymmetry of RF and GCM muscle activity during STS affects the step length asymmetry during walking in stroke patients.

The results of the present study showed positive correlation between RF asymmetry during STS and step time asymmetry during gait. Development of ipsilateral quadriceps weakness after stroke is associated with delayed recovery of function (Harris et al., 2001). Quadriceps weakness can decrease shock absorption capacity during walking, leading to accentuated symptoms and changes in gait patterns to maintain functionality (Clermont and Barden, 2016). Spinoso et al. (2018) reported that knee extensor strength is correlated with step time, and suggested that muscle weakness results in temporal changes in gait. Considering the present study's results, it seems that the asymmetry of RF muscle activity during STS affects the step time asymmetry during walking in stroke patients. This finding corresponds to those of previous study (Park et al., 2016) that the observed that gait pattern changes are associated with weakness of the anterior thigh muscles.

The results of the present study showed that FTSTS times were significantly negatively correlated with gait speed. The FTSTS times correlated with functional muscle strength of the lower limbs and dynamic standing balance of stroke patients ( $\mathrm{Ng}, 2010)$. Stroke patients who take less time to rise from sitting show significantly better gait velocity, cadence, and stride time (Chou et al., 2003). In addition, Chan et al. (2017) reported that FTSTS times are significantly correlated with Timed Up and Go test motor times. The result of present study found that a shorter FTSTS time is correlated with increased gait speed, consistent with previous findings.

The present study had some limitations. First, the sample size in this study was small. Second, we examined only a few lower limb muscles. Third, this study did not record kinetic data such as ground reaction force throughout the experiment. Future studies should include more muscle groups and collect kinetic data.

This study found a positive correlation between GCM and RF asymmetry during STS and step length, step time asymmetry during gait. FTSTS times were significantly negatively correlated with gait speed. Therefore, to improve step length and step time symmetry, it is necessary to apply exercises that focus on GCM and RF symmetry during STS. In addition, to improve gait speed, it is necessary to apply exercise that focuses on increase STS speed.

\section{CONFLICT OF INTEREST}

No potential conflict of interest relevant to this article was reported.

\section{REFERENCES}

Algurén B, Lundgren-Nilsson A, Sunnerhagen KS. Functioning of stroke survivors-a validation of the ICF core set for stroke in Sweden. Disabil Rehabil 2010;32:551-559.

Allen JL, Kautz SA, Neptune RR. Step length asymmetry is representative of compensatory mechanisms used in post-stroke hemiparetic walking. Gait Posture 2011;33:538-543. 
Bohannon RW. Knee extension strength and body weight determine sit-tostand independence after stroke. Physiother Theory Pract 2007;23:291297.

Bohannon RW, Barreca SR, Shove ME, Lambert C, Masters LM, Sigouin CS. Documentation of daily sit-to-stands performed by communitydwelling adults. Physiother Theory Pract 2008;24:437-442.

Chan PP, Si Tou JI, Tse MM, Ng SS. Reliability and validity of the Timed Up and Go test with a motor task in people with chronic stroke. Arch Phys Med Rehabil 2017;98:2213-2220.

Cheng PT, Liaw MY, Wong MK, Tang FT, Lee MY, Lin PS. The sit-to-stand movement in stroke patients and its correlation with falling. Arch Phys Med Rehabil 1998;79:1043-1046.

Chitra J, Mishra S. Effect of compelled body weight shift therapy on weight bearing symmetry and balance in post stroke patients: an experimental pre-post study. Int J Physiother Res 2014;2:781-786.

Chou SW, Wong AM, Leong CP, Hong WS, Tang FT, Lin TH. Postural control during sit-to stand and gait in stroke patients. Am J Phys Med Rehabil 2003;82:42-47.

Christiansen CL, Bade MJ, Weitzenkamp DA, Stevens-Lapsley JE. Factors predicting weight-bearing asymmetry 1 month after unilateral total knee arthroplasty: a cross-sectional study. Gait Posture 2013;37:363-367.

Clermont CA, Barden JM. Accelerometer-based determination of gait variability in older adults with knee osteoarthritis. Gait Posture 2016;50: 126-130.

de Sousa DG, Harvey LA, Dorsch S, Varettas B, Jamieson S, Murphy A, Giaccari $S$. Two weeks of intensive sit-to-stand training in addition to usual care improves sit-to-stand ability in people who are unable to stand up independently after stroke: a randomised trial. J Physiother 2019;65:152-158.

Geyh S, Cieza A, Schouten J, Dickson H, Frommelt P, Omar Z, Kostanjsek N, Ring H, Stucki G. ICF Core Sets for stroke. J Rehabil Med 2004;(44 Suppl):135-141.

Harris ML, Polkey MI, Bath PM, Moxham J. Quadriceps muscle weakness following acute hemiplegic stroke. Clin Rehabil 2001;15:274-281.

Hermens HJ, Freriks B, Disselhorst-Klug C, Rau G. Development of recommendations for SEMG sensors and sensor placement procedures. J Electromyogr Kinesiol 2000;10:361-374.

Hsu AL, Tang PF, Jan MH. Analysis of impairments influencing gait velocity and asymmetry of hemiplegic patients after mild to moderate stroke. Arch Phys Med Rehabil 2003;84:1185-1193.

Janssen W, Bussmann J, Selles R, Koudstaal P, Ribbers G, Stam H. Recovery of the sit-to-stand movement after stroke: a longitudinal cohort study. Neurorehabil Neural Repair 2010;24:763-769.

Khemlani MM, Carr JH, Crosbie WJ. Muscle synergies and joint linkages in sit-to-stand under two initial foot positions. Clin Biomech (Bristol,
Avon) 1999;14:236-246.

Lamontagne A, Malouin F, Richards CL, Dumas F. Mechanisms of disturbed motor control in ankle weakness during gait after stroke. Gait Posture 2002;15:244-255.

Lomaglio MJ, Eng JJ. Muscle strength and weight-bearing symmetry relate to sit-to-stand performance in individuals with stroke. Gait Posture 2005;22:126-131.

McCarthy EK, Horvat MA, Holtsberg PA, Wisenbaker JM. Repeated chair stands as a measure of lower limb strength in sexagenarian women. J Gerontol A Biol Sci Med Sci 2004;59:1207-1212.

Nasciutti-Prudente C, Oliveira FG, Houri SF, de Paula Goulart FR, Neto $\mathrm{MH}$, Teixeira-Salmela LF. Relationships between muscular torque and gait speed in chronic hemiparetic subjects. Disabil Rehabil 2009;31: 103-108.

$\mathrm{Ng}$ S. Balance ability, not muscle strength and exercise endurance, determines the performance of hemiparetic subjects on the timed-sit-tostand test. Am J Phys Med Rehabil 2010;89:497-504.

Park SK, Kobsar D, Ferber R. Relationship between lower limb muscle strength, self-reported pain and function, and frontal plane gait kinematics in knee osteoarthritis. Clin Biomech (Bristol, Avon) 2016;38:68-74.

Patterson KK, Gage WH, Brooks D, Black SE, Mcllroy WE. Evaluation of gait symmetry after stroke: a comparison of current methods and recommendations for standardization. Gait Posture 2010;31:241-246.

Prudente C, Rodrigues-de-Paula F, Faria CD. Lower limb muscle activation during the sit-to-stand task in subjects who have had a stroke. Am J Phys Med Rehabil 2013;92:666-675.

Pyöriä O, Era P, Talvitie U. Relationships between standing balance and symmetry measurements in patients following recent strokes (3 weeks or less) or older strokes (6 months or more). Phys Ther 2004;84:128-136.

Spinoso DH, Bellei NC, Marques NR, Navega MT. Quadriceps muscle weakness influences the gait pattern in women with knee osteoarthritis. Adv Rheumatol 2018;58:26.

Tomoyuki S, Shigeru U. Association of ability to rise from bed with improvement of functional limitation and activities of daily living in hemiplegic inpatients with stroke: a prospective cohort study. J Phys Ther Sci 2010;22:22-34.

van de Port IG, Kwakkel G, Lindeman E. Community ambulation in patients with chronic stroke: how is it related to gait speed? J Rehabil Med 2008;40:23-27.

Winstein CJ, Gardner ER, McNeal DR, Barto PS, Nicholson DE. Standing balance training: effect on balance and locomotion in hemiparetic adults. Arch Phys Med Rehabil 1989;70:755-762.

Yen SC, Schmit BD, Wu M. Using swing resistance and assistance to improve gait symmetry in individuals post-stroke. Hum Mov Sci 2015; 42:212-224. 